\title{
Unified gradient flow structure of phase field systems via a generalized principle of virtual powers
}

\author{
Elena Bonetti \\ Dipartimento di Matematica, Università degli Studi di Milano \\ Via Saldini 50, I-20133 Milano, Italy \\ E-mail: elena.bonetti@unimi.it \\ Elisabetta Rocca \\ Dipartimento di Matematica, Università degli Studi di Pavia \\ Via Ferrata 5, I-27100 Pavia, Italy \\ E-mail: elisabetta.rocca@unipv.it
}

June 29, 2016

\begin{abstract}
In this paper we introduce a general abstract formulation of a variational thermomechanical model by means of a unified derivation via a generalization of the principle of virtual powers for all the variables of the system, possibly including the thermal one. In particular, through a suitable choice of the driving functional, we formally get a gradient flow structure (in a suitable abstract setting) for the whole nonlinear PDE system. In this framework, the equations may be interpreted as internal balance equations of forces (e.g., thermal or mechanical ones). We prove a global in time existence of (a suitably defined weak) solutions for the Cauchy problem associated to the abstract PDE system as well as uniqueness in case of suitable smoothness assumptions on the functionals.
\end{abstract}

Key words: gradient flow, phase field systems, existence of weak solutions, uniqueness.

AMS (MOS) subject classification: 74N25, 82B26, 35A01, 35A02.

\section{Introduction}

In this paper we introduce a general derivation of thermo-mechanical phase transition models by use of a generalization of the principle of virtual powers, accounting for both micro-forces and thermal forces. It is known that a recent field of research in 
the framework of phase transitions has concerned models accounting for some microforces (see, e.g., the approaches by Frémond [13] and by Gurtin [16]). The main idea is that the equations governing the evolution of phase transition phenomena may be derived by a variational principle, the principle of virtual powers, in which microforces, responsible for phase transitions (i.e. for changes in the microstructure level of the materials) are included. As a consequence, the resulting PDE system provides an intrinsic variational structure, at least concerning equations for displacements and internal quantities, as phase or order parameters. Many authors have dealt with this kind of approach. We mention, among the others, contributions [19], [12], and [24].

On the other hand, as far as thermal properties are concerned, in the recent years several efforts have been spent to investigate models in which an entropy balance (or imbalance) equation was introduced in place of the more classical "heat equation". We recall, e.g., the contributions [3], [4], and [5]. In particular, let us mention that the last paper shows a derivation of the entropy equation by convex analysis tools and the application of a partial Legendre transformation for the free energy. It is interesting to observe that in this framework also thermal memory is formally justified from the point of view of the derivation of the model.

In a different direction Podio-Guidugli, in relation to a theory proposed by Green and Naghdi, introduced the possibility of including thermal displacements and forces in the whole balance of the principle of virtual powers, so that the entropy equation may be recovered, as well as the momentum equation, as a "balance of forces", forcing the system on the basis of some "reluctance to order".

Indeed, starting from the consideration that some virtual power principle may be exploited to deduce all balance and imbalance laws of thermomechanics, he suggested to use it also for the derivation of thermal evolution, introducing the notion of thermal displacement. As a consequence, he has derived an equation for the entropy of the system, which is combined with momentum balance. This approach turns out to be consistent with thermodynamical principles. See, among the others, contribution [26], the papers by Green and Naghdi [14], [15], and references therein for more details on the subject. Finally, we can quote some related recent contributions [22] and [20]. In [22] a gradient structure of systems in thermoplasticity is introduced by means of a free entropy functional instead of the internal energy, which is the driving functional in the present contribution. In [20] the author shows that many parabolic systems (like Allen-Cahn, Cahn-Hilliard, and reaction diffusion systems) can be written as gradient systems with respect to suitable functionals.

In this paper we aim to provide a generalized version of the principle of virtual powers leading to a variational abstract setting including, as applications, different phase transitions and phase separation models, accounting also for mechanical or thermal effects. Hence, we introduce an unified approach which formally justifies the evolution of the internal variables in terms of a gradient flow and the derivation of the balance equations for thermal and mechanical (micro or macro) variables is the same. The main advantage of the gradient structure is the possibility of deriving a time-incremental minimization procedure, where the internal energy functional is minimized with respect to the entropy and the internal variables. Consequently, the existence of weak solutions for the associated Cauchy problem can be deduced under 
quite general assumptions on the involved nonlinearities. Actually, in the following, we are dealing with two state variables: $s$, which can be interpreted (depending on the physical meaning of the equations) as a thermal variable (e.g., standing for the entropy, the internal energy or a function of the entropy), and an internal parameter $\chi$, representing the internal phase variable. Note that we have chosen here for the thermal variable the symbol $s$, which is a notation commonly used for the entropy, having in mind as a possible application of our general theory the so-called "entropy" models introduced, e.g., in $[3,4,26,11]$ where the standard internal energy balance is replaced by an entropy balance (cf. also. Section 2.3 where we introduce the "entropy model for phase transition" as possible example for our theory). However different interpretations are possible: for example $s$ can be interpreted as an internal energy variable and in this case the following equation (2.18) implies the conservation of total energy while the driving functional $e$ (2.13) could have the physical meaning of the entropy functional changed in sign. In this case the total entropy does not decrease during the evolution in agreement with the second law of Thermodynamics. This also the case of the other two examples we make as possible application of our theory in Section 2.3: the so-called Caginalp and Penrose-Fife phase-field models (cf. also [7] and [25]).

In our contribution the choice of the energy functional and the dissipation potential can be fairly general. In particular, in the internal energy functional we can include multivalued operators on both variables $s$ and $\chi$ to ensure some internal constraints. Since the resulting gradient flow structure is nonlinear and non smooth, we have to introduce a suitable notion of (weak) solution in order to get a global in time existence result. In a fairly general setting of assumptions, we are able to show that the evolution system admits an "admissible energy state", in the sense that the energy of the system is conserved, but for dissipative contributions. With some improved requirements we are able to prove the existence of a weak solution in a variational sense. However, the weak notion of solution we are introducing is naturally in accordance with the physical consistency of the problem. The proof is performed by means of a combined regularization and time discretization procedure. Moreover, uniqueness of solutions is proved under some further smoothness assumption on the internal energy functional.

The paper is organized as follows. In the next Section 2 we derive the model and state the main assumptions on the involved physical quantities and functionals. The main existence result is stated under the two sets of assumptions and proved in Section 3, as well as the uniqueness of solutions.

\section{The model and the main assumptions}

Let $\Omega \subset \mathbb{R}^{3}$ be a bounded and sufficiently regular domain with boundary $\Gamma:=\partial \Omega$. We introduce an Hilbert triplet $V \subset H \subset V^{\prime}$ (with dense and compact injections), where $V=H^{1}(\Omega), H=L^{2}(\Omega)$, and $H$ is identified as usual with its dual. We introduce the notations $\langle\cdot, \cdot\rangle$ for the duality pairing between $V$ and $V^{\prime}$ and $(\cdot, \cdot)$ for the usual scalar product both in $H$ and in $L^{2}(\Omega)^{3}$. To simplify the notation, we write $H$ in place of $L^{2}(\Omega)^{3}$, or $V$ in place of $H^{1}(\Omega)^{3}$, when vector-valued functions are considered. For 
every $f \in V^{\prime}$ we indicate by $\bar{f}$ the spatial mean of $f$ over $\Omega$, i.e.

$$
\bar{f}:=\frac{1}{|\Omega|}\langle f, 1\rangle
$$

where $|\Omega|$ stands for the Lebesgue measure of $\Omega$. We note as $H_{0}, V_{0}$ and $V_{0}^{\prime}$ the closed subspaces of functions (or functionals) having zero mean value in $H, V$, and in $V^{\prime}$, respectively. Then, by the Poincaré-Wirtinger inequality,

$$
\|v\|_{V_{0}}:=\left(\int_{\Omega}|\nabla v|^{2} \mathrm{~d} x\right)^{1 / 2}
$$

represents a norm on $V_{0}$ which is equivalent to the norm naturally inherited from $V$. In particular $\|\cdot\|_{V_{0}}$ is a Hilbert norm associated to a scalar product $((\cdot, \cdot))_{V_{0}}$ (defined in (2.1)), and thus we can introduce the associated Riesz isomorphism mapping $A$ : $V_{0} \rightarrow V_{0}^{\prime}$ by setting, for $u, v \in V_{0}$,

$$
\langle A u, v\rangle:=((u, v))_{V_{0}}:=\int_{\Omega} \nabla u \cdot \nabla v \mathrm{~d} x
$$

so that $\langle A u, u\rangle=\|u\|_{V_{0}}^{2}$ for every $u \in V_{0}$ and $\left\langle v, A^{-1}(v)\right\rangle=\|v\|_{V_{0}^{\prime}}^{2}$ for every $v \in V_{0}^{\prime}$. Finally, we can identify $H_{0}$ with $H_{0}^{\prime}$ by means of the scalar product of $H$ so to obtain the Hilbert triplet $V_{0} \subset H_{0} \subset V_{0}^{\prime}$, where inclusions are continuous and dense. In particular, if $z \in V$ and $v \in V_{0}$, it is easy to see that

$$
\int_{\Omega} \nabla z \cdot \nabla\left(A^{-1} v\right) \mathrm{d} x=\int_{\Omega}(z-\bar{z}) v \mathrm{~d} x=\int_{\Omega} z v \mathrm{~d} x .
$$

In what follows in this section we introduce our modelling approach and the set of PDEs and initial and boundary conditions which we are going to analyze in the next sections.

\subsection{The Principle of Virtual Powers}

The model is derived by using a variational principle in mechanics which is known as (generalized) principle of virtual power. Indeed, we refer to some generalization of the well known mechanical principle as we are including in the involved forces the microscopic forces, acting on some "micro-scale" (see [13]), and also possible "thermal forces". Without entering the details of this argumentation, let us point out that this principle is formally based on the fact that velocities are considered in a suitable linear space and thus forces are defined as elements acting on velocities with respect to some duality relation between the two spaces. This is done for any (sufficiently smooth) subdomain $\mathcal{D} \subseteq \Omega$. Hence, before proceeding we make precise the virtual velocities we are considering. We denote the couple of virtual velocities by $\left(\delta_{t}, v_{t}\right)$ (their physical meaning may change time to time). In the case when no accelerations are included, the principle of virtual powers can be written considering the power of internal forces $\mathcal{P}_{\text {int }}$ and of external forces $\mathcal{P}_{\text {ext }}$ (depending on $\left.\mathcal{D}, \delta_{t}, v_{t}\right)$ :

$$
\mathcal{P}_{\text {int }}\left(\mathcal{D}, \delta_{t}, v_{t}\right)+\mathcal{P}_{\text {ext }}\left(\mathcal{D}, \delta_{t}, v_{t}\right)=0 .
$$


We assume that the power of internal forces is introduced as follows (in $\Omega$ and for any virtual velocities $\delta_{t} \in V_{0}$ and $v_{t} \in V$ )

$$
\mathcal{P}_{\text {int }}=\left\langle\left\langle F, \delta_{t}\right\rangle\right\rangle+\left\langle\left\langle\mathcal{G}, v_{t}\right\rangle\right\rangle=\left\langle F, \delta_{t}\right\rangle+\int_{\Omega} B v_{t} \mathrm{~d} x+\int_{\Omega} E \nabla v_{t} \mathrm{~d} x
$$

where $F, B$ and $E$ denote interior thermal and mechanical (micro) forces and stresses, respectively and the duality relation $\langle\langle\cdot, \cdot\rangle\rangle$ is suitably defined between forces and velocities spaces.

Analogously, the power of external forces is

$$
\mathcal{P}_{\text {ext }}=\left\langle\left\langle\mathcal{R}, \delta_{t}\right\rangle\right\rangle+\left\langle\left\langle\mathcal{Z}, v_{t}\right\rangle\right\rangle .
$$

We let $v_{t} \in V$ and $\delta_{t} \in V_{0}$ and we assume there exists $Z, z$ such that

$$
\left\langle\left\langle\mathcal{R}, \delta_{t}\right\rangle\right\rangle=\left\langle\mathcal{R}, \delta_{t}\right\rangle \text { and }\left\langle\mathcal{Z}, v_{t}\right\rangle=\int_{\Omega} Z v_{t}+\int_{\Gamma} z v_{t},
$$

where $Z$ and $z$ stand for the external forces acting in the bulk $\Omega$ and at the boundary $\Gamma$, respectively.

It is clear that that we are considering a different behavior of the forces on the two types of virtual velocities. Indeed, we note that the elements $\mathcal{G}$ and $\mathcal{Z}$ are defined as a.e. forces living in the bulk and on the boundary (with suitable summability), while we take $F$ and $\mathcal{R}$ as general as possible to include all the different (and less regular) situations we will face. In particular, as it will be clear once we will make a precise choice of the actual velocities (cf. (2.5) and (2.6)), of the energy functional (2.13), and of the dissipation potential (2.14), we aim to write down an equation for the thermal variable of conservative type: it will result indeed as a conservation of energy, while the equation for the mechanical variable will be of non conservative type. This mainly motivates the choice we have made for the power of internal and external forces. Other choices are possible (cf., e.g., Remark 2.9), but we prefer not to move in this direction in the present contribution.

\subsection{The constitutive relations and the PDEs}

The state variables. We are dealing with a physical system governed by the state variables $(s, \chi, \nabla \chi)$ whose evolution is ruled by different thermomechanical relations. Note that we are distinguishing between different dependence of the energy with respect to the two variables $s$ and $\chi$ : we consider, in particular, the gradient $\nabla \chi$ but not $\nabla s$ as state variable (cf. (2.13)). This corresponds to the specific choice we have done for the forces $F, \mathcal{G}$ and $\mathcal{R}, \mathcal{Z}$ in (2.3) and (2.4).

In order to get the evolution of $s$, we take the actual velocities as $\delta_{t}=A^{-1}\left(\xi_{t}\right)$, where $\xi_{t} \in V_{0}^{\prime}$ and $v_{t}=0$, in order to get (for $\left.\mathcal{R}=0\right)\left\langle F, A^{-1}\left(\xi_{t}\right)\right\rangle=0$ for all $\xi_{t} \in V_{0}^{\prime}$ and so we obtain

$$
A(F)=0 \quad \text { in } V_{0}^{\prime} .
$$

The evolution of $\chi$ is obtained by integrating by parts in $\mathcal{P}_{\text {int }}$ and choosing $Z=z=0$ and $\delta_{t}=0$ as well:

$$
B-\operatorname{div} E=0 \quad \text { in } V^{\prime},
$$


with the no-mass flux through the boundary of $\Omega$ :

$$
E \cdot \mathbf{n}=0 \quad \text { on } \Gamma
$$

where we have denoted by $\mathbf{n}$ the outward unit normal vector to $\Gamma$. Notice that we have chosen here to have 0 external forces $\mathcal{R}$ and $\mathcal{Z}$ only for simplicity of notation.

Remark 2.1. In the following, we mainly refer to the variable $\chi$ as a phase or order parameter, i.e. related to the micro-structure of the physical system. However, let us point out that we could formally include in our procedure the derivation of the (more) classical momentum balance equation (letting, e.g., $\chi$ stand for displacements). In this case, the force $B$ has to be equal to 0 , due to the principle of rigid motions.

The functionals and the main assumptions. We introduce two functionals governing the evolution and the equilibrium of our (thermo)mechanical system. These functionals depend on the state variables and on the dissipative variables, respectively. As far as the equilibrium, it is governed by an energy functional, and we choose to make use of the internal energy functional (in place of the free energy functional). This choice is motivated by the fact that, in this framework, we may interpret $s$ as the entropy of the system (see [22] for a physical justification). However, it is well known that, under suitable assumptions, the internal energy may be introduced as the partial Legendre transformation of the free energy.

First, we introduce a function $j(\theta, \chi): \mathbb{R} \times \mathbb{R} \rightarrow[0,+\infty]$ such that

$$
\begin{aligned}
& \theta \mapsto j(\theta, \chi) \quad \text { is convex, proper, and lower semicontinuous for every } \chi \in \mathbb{R} \\
& \chi \mapsto-j(\theta, \chi) \quad \text { is convex, proper, and lower semicontinuous for every } \theta \in \mathbb{R},
\end{aligned}
$$

and let

$$
J_{H}(\theta, \chi)=\left\{\begin{array}{lll}
\int_{\Omega} j(\theta, \chi) & \text { if }(\theta, \chi) \in H \times H & \text { and } j(\theta, \chi) \in L^{1}(\Omega) \\
+\infty & \text { if }(\theta, \chi) \in H \times H & \text { and } j(\theta, \chi) \notin L^{1}(\Omega)
\end{array}\right.
$$

$$
J_{V}(\theta, \chi)=J_{H}(\theta, \chi) \quad \text { on } V \times V \text {. }
$$

Hence, we can introduce the convex partial conjugate of $J_{V}$ as follows $J_{V}^{*}(s, \chi)$ : $V^{\prime} \times V \rightarrow[0,+\infty]$ is defined as

$$
J_{V}^{*}(s, \chi)=\sup _{\theta \in V}\left(\langle s, \theta\rangle-J_{V}(\theta, \chi)\right), \quad(s, \chi) \in V^{\prime} \times V .
$$

Note that $J_{V}^{*}$ is a convex function with respect to $\chi$. Then, we can define the subdifferential (with respect to the variable $s$ ) $\partial_{V^{\prime}, V}^{s} J_{V}^{*}(s, \chi)$ which maps $V^{\prime} \times V$ into $2^{V}$ as (cf., e.g., [6]):

$$
\begin{array}{r}
\eta \in \partial_{V^{\prime}, V}^{s} J_{V}^{*}(s, \chi) \text { for } \chi \text { fixed, if and only if } \eta \in V,(s, \chi) \in D\left(J_{V}^{*}\right), \\
\text { and } J_{V}^{*}(s, \chi) \leqslant\langle s-w, \eta\rangle+J_{V}^{*}(w, \chi) \quad \forall w:(w, \chi) \in D\left(J_{V}^{*}\right) .
\end{array}
$$


In addition, we can define also the sub-differential of $J_{V}^{*}$ (with respect to the variable $\chi)$ mapping $V^{\prime} \times V$ into $2^{V^{\prime}}$, which is defined by

(2.12) $\xi \in \partial_{V, V^{\prime}}^{\chi} J_{V}^{*}(s, \chi)$ for $s$ fixed, if and only if $\xi \in V^{\prime}$,

$$
(s, \chi) \in D\left(J_{V}^{*}\right) \text { and } J_{V}^{*}(s, \chi) \leqslant\langle\xi, \chi-y\rangle+J_{V}^{*}(s, y) \quad \forall y:(s, y) \in D\left(J_{V}^{*}\right) .
$$

Actually, in what follows we will always work in the space $V^{\prime} \times V$ and so we will state directly the assumptions we need on the functional $J_{V}^{*}$ defined in (2.10). In particular, we prescribe the following assumptions:

Hypothesis 2.2. We assume that $J_{V}^{*}: V^{\prime} \times V \rightarrow[0,+\infty]$ is such that:

(J1) $s \mapsto J_{V}^{*}(s, \chi)$ is proper, convex and lower semicontinuous from $V^{\prime}$ to $[0,+\infty]$, for every $\chi \in V$,

(J2) $\chi \mapsto J_{V}^{*}(s, \chi)$ is proper, convex and lower semicontinuous from $V$ to $[0,+\infty]$, for every $s \in V^{\prime}$,

so that the subdifferentials $\partial_{V^{\prime}, V}^{s} J_{V}^{*}$ and $\partial_{V, V^{\prime}}^{\chi} J_{V}^{*}$, according to the definition (2.11) and (2.12), turn out to be maximal monotone operators acting from $V^{\prime}$ to $2^{V}$, for every $\chi \in V$, and from $V$ to $2^{V^{\prime}}$, for every $s \in V^{\prime}$, respectively(cf. [1]).

Hypothesis 2.3. Let us introduce a possibly non convex function $\hat{\gamma}$ depending on $\chi$ (w1) $\hat{\gamma}: \mathbb{R} \rightarrow \mathbb{R}$ is a $C^{1,1}$ function on $\mathbb{R}$.

In addition to Hyp. 2.2, to improve the existence result we are going to state, we need further assumptions on $J_{V}^{*}$ :

Hypothesis 2.4. The functional $J_{V}^{*}$ satisfies $J_{V}^{*}(s, \chi)=J_{1}^{*}(s)+J_{2}^{*}(\chi)+J_{3}^{*}(s-\chi)$, where $\theta \mapsto J_{3}^{*}(\theta)$ is convex, proper, l.s.c. from $V^{\prime}$ to $[0,+\infty]$ and one of the two sets of hypotheses holds

(A1) $s \mapsto J_{1}^{*}(s)$, is convex, proper, l.s.c. from $V^{\prime}$ to $[0,+\infty], \chi \mapsto J_{2}^{*}(\chi)$ is convex and l.s.c. from $V$ to $[0,+\infty)$ and the following bound holds: $\|\xi\|_{V^{\prime}} \leqslant c_{1}+c_{2}\|r\|_{V}$, $\forall r \in V, \xi \in \partial_{V, V^{\prime}} J_{2}^{*}(r) ; D\left(J_{1}^{*}\right) \cap \operatorname{Int} D\left(J_{3}^{*}\right) \neq \varnothing$

(A2) $\chi \mapsto J_{2}^{*}(\chi)$ is a proper, l.s.c. function from $V$ to $[0,+\infty]$ and $J_{1}^{*}(s)=0$; $D\left(J_{2}^{*}\right) \cap \operatorname{Int} D\left(J_{3}^{*}\right) \neq \varnothing$.

Now, we are in the position of introducing the internal energy functional $e$ : $V^{\prime} \times V \rightarrow(-\infty,+\infty]:$

$$
e(s, \chi)=J_{V}^{*}(s, \chi)+\int_{\Omega}\left(\frac{1}{2}|\nabla \chi|^{2}+\hat{\gamma}(\chi)\right) \mathrm{d} x .
$$

Let us note here that the first term in (2.13) contains both the purely caloric part of the energy functional (i.e. the one depending only on $s$ as well as the coupling terms 
depending on both $s$ and $\chi$ ) (cf. Subsection 2.3 for possible choices of $J_{V}^{*}$ ), while inside the integral over $\Omega$ we have the parts accounting for the nonlocal interfacial energy effects (the $|\nabla \chi|^{2}$ ) and the non-convex part of the mixing potential (cf. Remark 2.5). We intentionally choose not to consider interfacial (nonlocal) energy effects in the variable $s$ in order to differentiate the roles of the caloric and the mechanical parts $(s$ and $\chi$, respectively) in our approach.

Remark 2.5. Note that we can consider in our analysis the case in which the energy includes a term $W(\chi)=\widehat{\beta}(\chi)+\widehat{\gamma}(\chi)$ given by the sum of a convex possibly non-smooth part $\hat{\beta}$ (the term $J_{2}^{*}$ in $J_{V}^{*}$ ) and a non-convex but regular function $\hat{\gamma}$. Particularly meanigful choices of such mentioned $W$ used in the literature of phase transitions (when $\chi$ assumes the meaning of phase variable) are, for example,

1. the double well potential $W(\chi)=\left(\chi^{2}-1\right)^{2}$

2. the logarithmic potential $W(\chi)=\chi \log (\chi)+(1-\chi) \log (1-\chi)-\chi^{2}$

3. the double obstacle potential $W(\chi)=I_{[0,1]}(\chi)-\chi^{2}$, where $I_{[0,1]}$ denotes the indicator function of the interval $[0,1]$ and it is defined as $I_{[0,1]}(x)=0$ if $x \in[0,1]$ and $I_{[0,1]}(x)=+\infty$ otherwise.

Note that, the assumptions on $J_{V}^{*}$ follow from assumptions on $j$ and that possible examples of functions $j$ complying with our assumptions will be listed in the next Subsection 2.3. Actually, we could deal with non-convex functions, but in this case we should require further growth and smoothness conditions on the functional.

Remark 2.6. Observe that the assumptions on the positivity of the maps $j$ and $J_{V}^{*}$ could be weakened: we need indeed to have only a lower bound (possibly with a negative constant) for them in order to perform the first a-priori estimate (3.17).

We introduce as dissipative variables the time derivatives $s_{t}$ and $\chi_{t}$ and we include dissipation in the model by choosing the following form for the pseudo-potential of dissipation (see, e.g. [13], for a definition of the pseudo-potential of dissipation à la Moreau) depending on the dissipative variables $s_{t}$ and $\chi_{t}$. Note that we suppose the evolution to be rate dependent. The first possibility we consider for the dissipation functional is

$$
\varphi: V_{0}^{\prime} \times H \rightarrow \mathbb{R}, \quad \varphi\left(s_{t}, \chi_{t}\right)=\frac{1}{2}\left\langle s_{t}, A^{-1} s_{t}\right\rangle+\frac{1}{2} \int_{\Omega}\left|\chi_{t}\right|^{2} \mathrm{~d} x
$$

Note that we have a natural scalar product in $V_{0}^{\prime}((\cdot, \cdot))_{V_{0}^{\prime}}$ defined as $\left(\left(s_{t}, s_{t}\right)\right)_{V_{0}^{\prime}}=$ $\left\langle s_{t}, A^{-1} s_{t}\right\rangle=\left\|s_{t}\right\|_{V_{0}^{\prime}}^{2}, \int_{\Omega}\left|\chi_{t}\right|^{2} \mathrm{~d} x=\left(\chi_{t}, \chi_{t}\right)=\left\|\chi_{t}\right\|_{H}^{2}$. In the defintions of $e$ and $\varphi$ we have normalized all the physical constants to 1 for simplicity and without any loss of generality. Another possibility consists in letting

$$
\varphi\left(s_{t}, \chi_{t}\right)=\frac{1}{2}\left\langle s_{t}, A^{-1} s_{t}\right\rangle+\frac{1}{2}\left\langle\chi_{t}, A^{-1} \chi_{t}\right\rangle,
$$

but we prefer not to exploit this case in the present contribution in order to distinguish between the roles of the two variables: the thermal variable $s$ (conserved) and the 
mechanical variable $\chi$ (non conserved). Moreover, a rate-independent model could be introduced in place of the rate-dependent one we analyze here by suitably modifying the choice of the dissipation functional (2.14) (cf., e.g., [23], [10], and [21]). However, the analysis we are performing does not apply to this case, which would require ad hoc techniques and some suitable notion of weak solution.

The constitutive relations and the PDEs. Now, according to the definition of $\mathcal{P}_{\text {int }}$ and of $e$ and $\varphi$ (cf. (2.13) and (2.14)), we let the thermal force $F$ be

$$
F=\partial_{s} e+\partial_{s_{t}} \varphi=\partial_{V^{\prime}, V}^{s} J_{V}^{*}(s, \chi)+A^{-1}\left(s_{t}\right) .
$$

Hence, for the evolution of $\chi$ we prescribe the following mechanical (micro) forces and stresses $B$ and $E$ :

$$
B=\partial_{\chi} e+\partial_{\chi} \varphi=\partial_{V, V^{\prime}}^{\chi} J_{V}^{*}(s, \chi)+\hat{\gamma}^{\prime}(\chi)+\chi_{t}, \quad E=\partial_{\nabla \chi} e=\nabla \chi
$$

From (2.5-2.7) and the above constitutive relations we deduce the following PDE system for the evolution of $s$ and $\chi$ :

$$
\begin{aligned}
& s_{t}+A \eta=0 \text { in } V_{0}^{\prime}, \eta \in \partial_{V^{\prime}, V}^{s} J_{V}^{*}(s, \chi), \quad \text { a.e. in }(0, T), \\
& \chi_{t}+A \chi+\xi+\gamma(\chi)=0, \xi \in \partial_{V, V^{\prime}}^{\chi} J_{V}^{*}(s, \chi) \text { a.e. in }(0, T),
\end{aligned}
$$

where we denote by $\gamma=\hat{\gamma}^{\prime}$.

Notice that system (2.18)-(2.19) can be rewritten in terms of the vector $u:=$ $(s, \chi)$ in a more general framework, as the gradient-flow associated to the functional

$$
\Phi(u)=\Phi(s, \chi)=J_{V}^{*}(s, \chi)+\int_{\Omega}\left(\frac{1}{2}|\nabla \chi|^{2}+\widehat{\gamma}(\chi)\right) \mathrm{d} x,
$$

as

$$
\mathcal{N}\left(u_{t}\right)+\frac{\delta \Phi}{\delta u} \ni 0 \quad \text { in }(0, T)
$$

where $\mathcal{N}$ is the duality map between $\mathcal{H}:=V_{0}^{\prime} \times H$ and $V_{0} \times H$ induced by the norm

$$
\|u\|_{\mathcal{H}}^{2}:=\left\langle A^{-1}(s), s\right\rangle+\int_{\Omega}|\chi|^{2} \mathrm{~d} x, \quad \text { so that } \mathcal{N}(s, \chi):=\left(A^{-1}(s), \chi\right) .
$$

Remark 2.7. Let us notice that in case $\operatorname{dom}(j)=\mathbb{R}$, which is also equivalent to assume

$$
\lim _{|r| \rightarrow+\infty} \frac{j^{*}(r)}{|r|}=+\infty
$$

then we can prove that (cf. [2]) the functional e defined in (2.13) can be rewritten as

$$
e(s, \chi)=\int_{\Omega}\left(j^{*}(s, \chi)+\frac{1}{2}|\nabla \chi|^{2}+\widehat{\gamma}(\chi)\right) \mathrm{d} x
$$


where $j^{*}$ is the conjugate function of $j$ with respect to the variable $s$, i.e.

$$
j^{*}(s, \chi)=\sup _{\theta \in \mathbb{R}}(s \theta-j(\theta, \chi)), \quad \forall(s, \chi) \in \mathbb{R} \times \mathbb{R} .
$$

Moreover, the inclusion (2.18) can be rewritten as the following gradient flow in $V_{0}^{\prime}$ :

$$
s_{t}+\partial_{V^{\prime}} J_{V}^{*}(s, \chi) \ni 0
$$

where $\partial_{V^{\prime}} J_{V}^{*}$ is defined as the subdifferential of $J_{V}^{*}$ in $V^{\prime}$ mapping $V^{\prime} \times V$ into $2^{V^{\prime}}$ as follows:

$$
\begin{aligned}
& \xi \in \partial_{V^{\prime}} J_{V}^{*}(s, \chi) \quad \text { if and only if } \xi \in V^{\prime}, \\
& \quad(s, \chi) \in D\left(J_{V}^{*}\right), \text { and } J_{V}^{*}(s, \chi) \leqslant((s-w, \xi))_{*}+J_{V}^{*}(w, \chi) \quad \forall(w, \chi) \in V^{\prime} \times V,
\end{aligned}
$$

where $((\cdot, \cdot))_{*}$ denotes the scalar product in $V^{\prime}$. The reader can refer to [1] and to $[2$, Section 2] for the proofs of these results. Finally in this case we have $u \in \partial_{V^{\prime}, V}^{s} J_{V}(s, \chi)$ in $V$ if and only if $u \in \partial_{s} j^{*}(s, \chi)$ a.e. in $\Omega$, where $\partial_{s}$ denotes here the subdifferential of convex analysis with respect to the variable $s$ (cf., e.g., [6]).

\subsection{Possible choices of $j^{*}$}

In this section we show how to derive different types of phase-field models by our general system.

The Caginalp model of phase transitions. Choose $j^{*}(s, \chi)=\frac{s^{2}}{2}-s \chi+\frac{\chi^{2}}{2}+\widehat{\beta}(\chi)$, with $\hat{\beta}$ a proper, convex, s.c.i. function (cf. Remark 2.5 for possible examples of $\widehat{\beta}$ ). Denote by $\theta:=\partial_{s} j^{*}=s-\chi$ and by $\beta=\partial \widehat{\beta}$. Then, the PDEs $(2.18-2.19)$ can be rewritten as

$$
\begin{aligned}
& \theta_{t}+\chi_{t}-\Delta \theta=0, \\
& \chi_{t}-\Delta \chi+\beta(\chi)+\gamma(\chi)-\theta \ni 0,
\end{aligned}
$$

coupled with Neumann homogeneous boundary conditions on $\theta$ and $\chi$. This PDE system can be easily identified with the "standard" phase field model of Caginalp type (cf. [7]), letting $\theta$ be the relative temperature of the system and $\chi$ the local proportion of one of the two phases of the substance undergoing phase transitions. Here Hyp. 2.2 and 2.4 (A2) are satisfied with the choices $J_{1}^{*}=0, J_{2}^{*}$ coming from $j_{2}^{*}(\chi)=\widehat{\beta}(\chi), J_{3}^{*}$ defined starting from $j_{3}^{*}(s-\chi)=(s-\chi)^{2} / 2$.

The entropy model for phase transitions. Choosing $j^{*}(s, \chi)=\exp (s-\chi)+\widehat{\beta}(\chi)$, for $\hat{\beta}$ a proper, convex, s.c.i. function, we have that Hyp. 2.2 is satisfied. Actually, we point out that we could extend our analysis to the case in which $\chi$ is substituted by a function $\lambda(\chi)$ which is a Lipschitz continuous function on the domain of $\beta=\partial \widehat{\beta}$. In this case Hyp. 2.4 is satisfied with the choices $j_{1}^{*}=0, j_{2}^{*}(\chi)=\widehat{\beta}(\chi), j_{3}^{*}(s-\chi)=\exp (s-\chi)$. 
Then, defining $\theta:=\partial_{s} j^{*}=\exp (s-\lambda(\chi))$, we get $s=\log \theta+\chi$ and the PDEs (2.18-2.19) can be rewritted as

$$
\begin{aligned}
& (\log \theta+\chi)_{t}-\Delta \theta=0, \\
& \chi_{t}-\Delta \chi+\beta(\chi)+\gamma(\chi)-\theta \ni 0,
\end{aligned}
$$

again with Neumann homogeneous boundary conditions for both $\theta$ and $\chi$. This system can be easily identified with the "entropy" phase field model introduced in [3] and [4].

The Penrose-Fife model for phase transitions. We choose $j^{*}(s, \chi)=-\log (s-$ $\chi)+\hat{\beta}(\chi)$, for $s>\chi$ and define $\frac{1}{\theta}:=-\partial_{s} j^{*}(s, \chi)=\frac{1}{s-\chi}$. Then, we observe that we can formally get the Penrose-Fife model, where here $\theta$ plays the role of the absolute temperature (see [25] for a detailed description of the model). Indeed, it results that $\partial_{\chi} j^{*}(s, \chi)=\frac{1}{s-\chi}+\beta(\chi)=\frac{1}{\theta}+\beta(\chi)$. Thus, we can rewrite $(2.18-2.19)$ as

$$
\begin{aligned}
& (\theta+\chi)_{t}-\Delta\left(-\frac{1}{\theta}\right)=0, \\
& \chi_{t}-\Delta \chi+\beta(\chi)+\gamma(\chi)+\frac{1}{\theta} \ni 0,
\end{aligned}
$$

coupled with Neumann homogeneous boundary conditions on $-\frac{1}{\theta}$ and $\chi$. Let us notice that in this case the assumption $D\left(j^{*}\right)=\mathbb{R}$ is not verified, hence we are not entitled to use the function $j^{*}$ instead of the operator $J_{V}^{*}$ in $e$, so, the choice we made here is only formal. For a rigorous analysis of this case the reader can refer to [30]. Here Hyp. 2.4 (A2) is satisfied with the choices: $j_{1}^{*}=0, j_{2}^{*}(\chi)=\widehat{\beta}(\chi)$ (cf. Remark 2.5), $j_{3}^{*}(s-\chi)=-\log (s-\chi)$.

Remark 2.8. Another related example we could possibly deal with is a simplification of the tumor growth model recently introduced in $[17,18]$. Indeed, choosing $s=\varphi$, standing for the tumor phase, and $\chi=\sigma$, representing the nutrient concentration, we could write down a simplified version of the model introduced in [17, 18] as the following gradient flow system (cf. [27]):

$$
\left(\varphi_{t}, \sigma_{t}\right)=-\nabla_{X \times Y} E(\varphi, \sigma),
$$

where $X \times Y=V_{0}^{\prime} \times H$, being the energy functional $E$ defined as

$$
E(\varphi, \sigma):= \begin{cases}M(\varphi-\sigma)+F(\varphi, \sigma) & \text { if }(\varphi, \sigma) \in H \times V, \varphi-\sigma \in V \\ +\infty & \text { otherwise. }\end{cases}
$$

Here $M$ is the functional defined on $V$ by

$$
M(u):=\int_{\Omega}\left(\frac{W(u)}{\varepsilon}+\frac{\varepsilon}{2}|\nabla u|^{2}\right) d x
$$

and $F$ is the function on $H \times V$ into $L^{1}(\Omega)$ given by

$$
F(\varphi, \sigma):=\int_{\Omega}\left(\frac{|\varphi-\sigma|^{2}}{2}+\frac{|\nabla \sigma|^{2}}{2}\right) d x .
$$


This case could fit the Hyp. 2.4 with the choices $J_{1}^{*}(\varphi)=0, J_{2}^{*}(\sigma)=0, J_{3}^{*}(\varphi-\sigma)$ defined from $j_{3}^{*}(u)=\frac{W(u)}{\varepsilon}+\frac{|u|^{2}}{2}$, but we would need to add a term of the type $\int_{\Omega} \frac{\varepsilon}{2}|\nabla(\varphi-\sigma)|^{2} d x$ in the internal energy $e$ and to require that the term $W$ to be convex in this case. However, this could be considered in further developments of our analysis.

Remark 2.9. Let us notice that in case we choose as pseudopotential of dissipation the functional (2.15) in (2.16) and (2.17), the first equation is the same as (2.18), while the equation for $\chi$ results as:

$$
A^{-1} \chi_{t}-\Delta \chi+\gamma(\chi)+\partial_{\chi} j^{*}(s, \chi) \ni 0
$$

and thus

$$
\begin{aligned}
& s_{t}+A\left(\partial_{s} j^{*}(s, \chi)\right) \ni 0 \text { in } V_{0}^{\prime}, \\
& \chi_{t}+A w=0, \quad w \in-\Delta \chi+\gamma(\chi)+\partial_{\chi} j^{*}(s, \chi),
\end{aligned}
$$

coupled with Neumann homogeneous boundary conditions for $\partial_{s} j^{*}(s, \chi), \chi$ and $w$. In this case the evolution of $\chi$ is ruled by the well-known fourth order Cahn-Hilliard equation modelling phase separation phenomena (cf., e.g., [8]). However, as we already mentioned, we prefer not to deal with this case here.

\section{Main results}

In this section we state the main results of this paper, the first one (Thm. 3.1) concerns the existence of global in time of "admissible energy state" and weak solutions for system (2.18)-(2.19) coupled with the boundary condition (2.7) and the initial conditions

$$
\begin{aligned}
& s(0)=s_{0} \quad \text { in } V_{0}^{\prime}, \\
& \chi(0)=\chi_{0} \quad \text { a.e. in } \Omega,
\end{aligned}
$$

while the second one (Thm. 3.4) regards uniqueness of solutions under more restrictive assumptions on the nonlinearities involved.

In the statement of Theorem 3.1 we distinguish between two sets of hypotheses, leading to different notion of weak solutions.

Theorem 3.1. Assume Hypotheses 2.2, 2.3, and take $\left(s_{0}, \chi_{0}\right) \in D\left(J_{V}^{*}\right)$. Then, for every $T>0$ there exists at least a couple $(s, \chi)$ with regularity

$$
\begin{aligned}
& s \in H^{1}\left(0, T ; V_{0}^{\prime}\right), \\
& \chi \in H^{1}(0, T ; H) \cap L^{\infty}(0, T ; V)
\end{aligned}
$$

fulfilling

$$
\int_{0}^{t}\left(\left\|s_{t}\right\|_{V_{0}^{\prime}}^{2}+\left\|\chi_{t}\right\|_{H}^{2}\right)+\Phi(s(t), \chi(t)) \leqslant \Phi\left(s_{0}, \chi_{0}\right)
$$


where $\Phi$ is defined in (2.20). Such a couple satisfying (3.5) is called "admissible energy state" to problem (2.18)-(2.19) and (3.1)-(3.2).

If in addition we assume that Hyp. $2.4(A 1)$ or $(A 2)$ with $s_{0} \in H$ hold true, then there exists at least a couple $(s, \chi)$ with the regularity (3.3-3.4) satisfying (2.18)(2.19) and (3.1)-(3.2), and (3.5). In particular, if $(A 2)$ is prescribed and $s_{0} \in H$, then the solution fulfills

$$
s \in L^{\infty}(0, T ; H) .
$$

It is clear that a weak solution is also an admissible energy state for the system.

Proof. In order to prove Theorem 3.1, we first approximate system (2.18-2.19) with a regularized problem depending on a positive small parameter $\varepsilon$ and then we pass to the limit by (weak-strong) compactness arguments and semicontinuity results based on sufficient a-priori estimates - independent of $\varepsilon$ - we are going to prove on the approximating solutions.

The approximated problem. Let us fix $\varepsilon>0$. Then, for every $T>0$ and $\left(s_{0, \varepsilon}, \chi_{0}\right) \in D\left(J_{V}^{*}\right) \cap(H \times V)$, we aim to find a solution $\left(s_{\varepsilon}, \chi_{\varepsilon}\right) \in H^{1}\left(0, T ; V_{0}^{\prime} \times H\right)$ to the following differential inclusions:

(3.6) $\partial_{t} s_{\varepsilon}+A\left(\eta_{\varepsilon}+\varepsilon s_{\varepsilon}\right)=0$ in $V_{0}^{\prime}, \eta_{\varepsilon} \in \partial_{V^{\prime}, V}^{s} J_{V}^{*}\left(s_{\varepsilon}, \chi_{\varepsilon}\right)$, for a.e. $t \in(0, T)$,

(3.7) $\partial_{t} \chi_{\varepsilon}+A \chi_{\varepsilon}+\xi_{\varepsilon}+\gamma\left(\chi_{\varepsilon}\right)=0$ in $V^{\prime}, \quad \xi_{\varepsilon} \in \partial_{V, V^{\prime}}^{\chi} J_{V}^{*}\left(s_{\varepsilon}, \chi_{\varepsilon}\right), \quad$ for a.e. $t \in(0, T)$,

coupled with the initial conditions (3.1-3.2), with $s_{0, \varepsilon}$ in place of $s_{0}$. In particular, we assume that

$$
s_{0, \varepsilon} \in H, \quad s_{0, \varepsilon} \rightarrow s_{0} \quad \text { in } V_{0}^{\prime} \quad \text { as } \varepsilon \searrow 0 .
$$

We first observe that we can recover (3.6) and and (3.7), by approximating the energy functional (2.20) as follows:

$$
\Phi_{\varepsilon}(s, \chi):=\Psi_{\varepsilon}(s, \chi)+\int_{\Omega}\left(\frac{1}{2}|\nabla \chi|^{2}+\hat{\gamma}(\chi)\right) \mathrm{d} x, \Psi_{\varepsilon}(s, \chi):=J_{V}^{*}(s, \chi)+\frac{\varepsilon}{2} \int_{\Omega}|s|^{2} \mathrm{~d} x .
$$

Actually, note that now $\Phi_{\varepsilon}$ is defined in $\left(V_{0}^{\prime} \cap H\right) \times V$. Hence, we can construct its subdifferential in the duality between $V_{0}^{\prime}$ and $V_{0}$, and rewrite the equation (3.6) as (cf. the definition (2.11)):

$$
\partial_{t} s_{\varepsilon}+A \zeta_{\varepsilon}=0 \text { in } V_{0}^{\prime}, \zeta_{\varepsilon} \in \partial_{V^{\prime}, V}^{s} \Phi_{\varepsilon}\left(s_{\varepsilon}, \chi_{\varepsilon}\right) \quad \text { for a.e. } t \in(0, T) \text {. }
$$

Now, our aim is to prove the existence of solutions of (3.6)-(3.7), (3.1)-(3.2) with $s_{0, \varepsilon}$ instead of $s_{0}$ by use of a time-discrete approximation, as follows (cf. also [28] for a similar procedure). Here we drop the index $\varepsilon$ in order to simplify the notation. Let us fix a time step $\tau=T / N, N \in \mathbb{N}$ and introduce a uniform partition

$$
P_{\tau}:=\left\{t_{0}=0, t_{1}=\tau, \ldots, t_{n}=n \tau, \ldots, t_{N}=T\right\}
$$


of the interval $(0, T)$. Then, we need to find a discrete approximation $s^{n} \sim s\left(t_{n}\right)$, $\chi^{n} \sim \chi\left(t_{n}\right)$ by solving the implicit Euler scheme (cf. also (2.21)):

$$
\mathcal{N}\left(\frac{U^{n}-U^{n-1}}{\tau}\right)+\zeta^{n}=0, \quad n=1, \ldots, N ; U^{0}:=u_{0},
$$

where $\zeta^{n} \in \frac{\delta \Phi_{\varepsilon}}{\delta u}\left(U^{n}\right)$ and we have defined $U^{n}=\left(s^{n}, \chi^{n}\right), u_{0}=\left(s_{0, \varepsilon}, \chi_{0}\right)$. Using the functional space, we have already introduced to define the operator $\mathcal{N}, \mathcal{H}=\left(V_{0}^{\prime} \cap\right.$ $H) \times V$, we notice that (3.10) is the Euler equation for the variational problem

$$
\left\{\begin{array}{l}
\text { find } U^{n} \in \mathcal{H} \text { minimizing } \\
F_{\varepsilon}\left(\tau, U^{n-1} ; U\right):=\frac{1}{2 \tau}\left\|U-U^{n-1}\right\|_{\mathcal{H}}^{2}+\Phi_{\varepsilon}(U), \quad U \in \mathcal{H} .
\end{array}\right.
$$

It is not difficult to see that this minimization problem is solvable due to the lowersemicontinuity and coercivity properties of $\Phi_{\varepsilon}$ (cf., e.g., [28, 29] and references therein for a similar variational approach to find a discrete solution).

Then, we can construct the piecewise constant interpolants $\bar{U}_{\tau}(t):=U^{n}$ if $t \in((n-1) \tau, n \tau]$. In particular, we get that and we recover the solution $U:=(s, \chi)$ of (3.6-3.7) as the limit of $\bar{U}_{\tau}$ as $\tau \searrow 0$. This can be done, by using suitable a priori estimates (independent of $\tau$ and then passing to the limit by compactness and semicontinuity arguments. We do not enter the details of the proof, as it is very similar to the estimates and passage to the limit procedure we are going to detail in the next sections to pass to the limit as $\varepsilon \searrow 0$. Note that in this case some technicalities are avoided due to the more regular setting for the variable $s_{\varepsilon}$ (recall the strict positivity of $\varepsilon$ ). Moreover, here we do not need to use the additional Hyp. 2.4 in order to prove the existence of weak variational solutions to (3.6)-(3.7) due to the fact that we are in the position of directly deduce the enhanced estimate (depending on $\varepsilon$ but not on $\tau)$ on $s_{\varepsilon}$ in $L^{\infty}\left(0, T ; V_{0}\right)$ which allows us to get strong convergence of the time discrete interpolant to $s$ as $\tau \searrow 0$ in $L^{\infty}(0, T ; H)$. With this we can pass to the limit in the subdifferentials as we will do it in the passage to the limit as $\varepsilon \searrow 0$ in case (A2) holds true (cf. (3.27)). Thus, we can easily prove the following existence result.

Theorem 3.2. Under the same assumptions of Theorem 3.1, letting $\varepsilon>0$ be fixed and (3.8) holds, then there exists a solution to (3.6)-(3.7) with $s_{\varepsilon}(0)=s_{0, \varepsilon}$ and $\chi_{\varepsilon}(0)=\chi_{0}$, with the following regularity

$$
\begin{aligned}
& s_{\varepsilon} \in H^{1}\left(0, T ; V_{0}^{\prime}\right) \cap L^{\infty}\left(0, T ; V_{0}\right), \\
& \chi_{\varepsilon} \in H^{1}(0, T ; H) \cap L^{\infty}(0, T ; V) .
\end{aligned}
$$

A priori estimates (uniform in $\varepsilon$ ). Let us consider the system (3.6-3.7), where for the sake of simplifying notation we neglect the index $\varepsilon$ for solutions and involved functions. We now perform the a priori estimates independent of $\varepsilon$, so, we use here the same symbol $c$ for positive constants, possibily different from line to line, depending on the problem data, but independent of $\varepsilon$.

In order to perform the first estimate we need to prove here a small variant of the chain rule formula stated, e.g., in [9, Prop. 4.2]. 
Proposition 3.3. Let $G: V^{\prime} \times V \rightarrow[0,+\infty]$ be a map such that $v \mapsto G(u, v)$ is a proper convex lower semicontinuous mapping for every $u \in V^{\prime}$, $u \mapsto G(u, v)$ is a proper convex lower semicontinuous mapping for every $v \in V$, and let $u \in H^{1}\left(0, T ; V^{\prime}\right) \cap L^{2}\left(0, T ; V_{0}\right), v \in H^{1}(0, T ; V) \cap L^{2}(0, T ; V)$, and $\delta(t) \in$ $\partial_{V^{\prime}, V}^{u} G(u(t), v(t))$ for a.e. $t \in(0, T)$, where the subdifferential $\partial_{V^{\prime}, V}^{u}$ is defined as in (2.11), and $\sigma(t) \in \partial_{V, V^{\prime}}^{v} G(u, v)$ defined by (2.12). Then the function $g=G(u(\cdot), v(\cdot))$ is absolutely continuous in $[0, T]$ and $g^{\prime}(t)=\left\langle u^{\prime}(t), \delta(t)\right\rangle+\left\langle\sigma(t), v^{\prime}(t)\right\rangle$ for a.e. $t \in(0, T)$.

Proof. Here we follow the lines of the proof of [9, Prop. 4.2]. Let $w \in$ $W^{1, \infty}(0, T)$ be a non-negative function with compact support in $(0, T)$. We choose $h>0$ such that $\operatorname{supp}(w) \subset[h, T-h]$. For a.e. $t \in(0, T)$, by assumptions we can infer that

$$
\begin{aligned}
& \langle u(t)-u(t-h), \delta(t-h)\rangle+\langle\sigma(t-h), v(t)-v(t-h)\rangle \\
& \leqslant g(t)-g(t-h) \leqslant\langle u(t)-u(t-h), \delta(t)\rangle+\langle\sigma(t), v(t)-v(t-h)\rangle .
\end{aligned}
$$

Indeed, observe that $\delta \in \partial_{V^{\prime}, V}^{u} G(u, v)$, i.e. to the sub-differential of the function $G: V^{\prime} \times V \rightarrow[0,+\infty]$ defined w.r.t. the variable $u$ and $\sigma \in \partial_{V, V^{\prime}}^{v} G(u, v)$, i.e. to the sub differential of $G$ w.r.t. $v$. Observe that we can extend $w$ outside of $(0, T)$ with the 0 value. Hence, multiplying by $w(t)$, integrating with respect to $t$, and letting $h \searrow 0$, we obtain

$$
\begin{aligned}
\frac{1}{h} \int_{h}^{T}\langle u(t)-u(t-h), \delta(t-h)\rangle w(t) d t & =\frac{1}{h} \int_{0}^{T-h}\langle u(t+h)-u(t), \delta(t)\rangle w(t+h) d t \\
& \rightarrow \int_{0}^{T}\left\langle u^{\prime}(t), \delta(t)\right\rangle w(t) d t \\
\frac{1}{h} \int_{h}^{T}(g(t)-g(t-h)) w(t) d t & =\frac{1}{h} \int_{0}^{T} \delta(t)(w(t)-w(t+h)) d t \\
& \rightarrow-\int_{0}^{T} \delta(t) w^{\prime}(t) d t, \\
\frac{1}{h} \int_{h}^{T}\langle u(t)-u(t-h), \delta(t)\rangle w(t) d t & \rightarrow \int_{0}^{T}\left\langle u^{\prime}(t), \delta(t)\right\rangle w(t) d t .
\end{aligned}
$$

Moreover, we can use the same argument for the term involving the variable $v$, so that at the end we conclude that

$$
-\int_{0}^{T} g(t) w^{\prime}(t) d t=\int_{0}^{T}\left(\left\langle u^{\prime}(t), \delta(t)\right\rangle+\left\langle\sigma(t), v^{\prime}(t)\right\rangle\right) w(t) d t
$$

for all non-negative Lipschitz continuous test functions $w$ with compact support. Since both the positive and the negative part of a Lipschitz continuous function are Lipschitz continuous, we obtain the assertion. 
First a priori estimate. We test (3.6) by $A^{-1} s_{t}$ using the defintion of the $V_{0}^{\prime}$ norm we have

$$
\left\langle s_{t}, A^{-1} s_{t}\right\rangle=\left\|s_{t}\right\|_{V_{0}^{\prime}}^{2},
$$

and in addition, using the definition of $J_{V}^{*}$ and a variant of the chain rule formula stated in Proposition 3.3 with the choices $G=J_{V}^{*}, u=s, v=\chi$, we get

$$
\begin{aligned}
\left\langle A(\eta+\varepsilon s), A^{-1} s_{t}\right\rangle & =\left\langle s_{t}, \eta\right\rangle+\varepsilon\left(s_{t}, s\right) \\
& =\frac{d}{d t}\left(J_{V}^{*}(s(t), \chi(t))+\frac{\varepsilon\|s(t)\|_{H}^{2}}{2}\right)-\left\langle\partial_{V, V^{\prime}}^{\chi} J_{V}^{*}(s, \chi), \chi_{t}\right\rangle .
\end{aligned}
$$

Testing (3.7) by $\chi_{t}$, we get

$$
\left\|\chi_{t}\right\|_{H}^{2}+\frac{1}{2} \frac{d}{d t}\|\nabla \chi\|_{H}^{2}+\frac{d}{d t} \int_{\Omega} \hat{\gamma}(\chi)+\left\langle\partial_{V, V^{\prime}}^{\chi} J_{V}^{*}(s, \chi), \chi_{t}\right\rangle=0 .
$$

Adding the resulting equations and integrating over $(0, t), t \in(0, T)$, and using the definition of $\Psi_{\varepsilon}$, we obtain

$$
\int_{0}^{t}\left(\left\|s_{t}\right\|_{V_{0}^{\prime}}^{2}+\left\|\chi_{t}\right\|_{H}^{2}\right) \mathrm{d} \tau+\Psi_{\varepsilon}(s(t), \chi(t))+\int_{\Omega} \widehat{\gamma}(\chi(t))+\|\nabla \chi(t)\|^{2} \leqslant c
$$

where here $c$ depends in particular on the initial data.

Here the tests we are making are formal and should be performed on the time discrete scheme because $\chi_{t}$ does not belong to $V$, which we would need here. However, we could proceed rigorously, e.g. by performing the estimate at a discrete level (note that the estimate should be rigorous because then $\chi_{t}$ is replaced by the differences of $\chi$ 's which belong to $V$ ) or performing the estimate approximating the graph by its Yosida regularization.

Adding to both sides in (3.17)

$$
\|\chi(t)\|_{H}^{2}=\left\|\chi_{0}\right\|_{H}^{2}+2 \int_{0}^{t}\left(\chi(\tau), \chi_{t}(\tau)\right) \mathrm{d} \tau
$$

and using Hölder and Young inequalities together with Hyp. 2.3 and 2.2 and a standard Gronwall lemma, we obtain

$$
\begin{aligned}
& \left\|s_{t}\right\|_{L^{2}\left(0, T ; V_{0}^{\prime}\right)}^{2}+\varepsilon\|s\|_{L^{\infty}(0, T ; H)}^{2} \leqslant c, \\
& \|\chi\|_{H^{1}(0, T ; H) \cap L^{\infty}(0, T ; V)} \leqslant c .
\end{aligned}
$$

Second a priori estimate. We proceed by a comparison in (3.9). Due to $(3.18)_{1}$, we have that $A \zeta$ is bounded in $L^{2}\left(0, T ; V_{0}^{\prime}\right)$, and thus

$$
\|\zeta\|_{L^{2}\left(0, T ; V_{0}\right)} \leqslant c .
$$

Hence, using (3.18), we get

$$
\|\eta+\varepsilon s\|_{L^{2}(0, T ; V)} \leqslant c,
$$


and so from $(3.18)_{2}$ it follows

$$
\|\eta\|_{L^{2}(0, T ; H)} \leqslant c .
$$

Indeed, due to the definition of $\Psi_{\varepsilon}$ we can infer that $\zeta_{\varepsilon}=\eta_{\varepsilon}+\partial \psi_{\varepsilon}(s)$, where we have used the notation $\psi_{\varepsilon}(s)=\frac{\varepsilon}{2} \int_{\Omega} s^{2} \mathrm{~d} x$ and the fact that the domain of $\psi_{\varepsilon}$ is the whole real line. Thus, its subdifferential in the duality $V^{\prime}, V$ corresponds to the standard subdifferential of the convex analysis (cf. [6]).

Third a priori estimate. By a comparison in (3.7), exploiting (3.19), we can deduce that

$$
\|\xi\|_{L^{2}\left(0, T ; V^{\prime}\right)} \leqslant c
$$

Passage to the limit as $\varepsilon \searrow 0$. Now, we aim to pass to the limit in (3.6)(3.7) as $\varepsilon \searrow 0$, recovering a solution to (2.18-2.19). By virtue of (3.18-3.22) and by compactness results we get (at least for some subsequences of $\varepsilon \searrow 0$ we still denote by $\varepsilon$ to simplify notation):

$$
\begin{aligned}
& s_{\varepsilon} \rightarrow s \quad \text { in } H^{1}\left(0, T ; V_{0}^{\prime}\right), \\
& \chi_{\varepsilon} \stackrel{*}{\rightarrow} \chi \quad \text { in } H^{1}(0, T ; H) \cap L^{\infty}(0, T ; V), \\
& \zeta_{\varepsilon} \rightarrow \zeta \quad \text { in } L^{2}\left(0, T ; V_{0}\right), \\
& \eta_{\varepsilon} \rightarrow \eta \quad \text { in } L^{2}(0, T ; H), \\
& \varepsilon^{1 / 2} s_{\varepsilon} \stackrel{*}{\rightarrow} 0 \quad \text { in } L^{\infty}(0, T ; H), \\
& \xi_{\varepsilon} \rightarrow \xi \quad \text { in } L^{2}\left(0, T ; V^{\prime}\right) .
\end{aligned}
$$

Notice that (3.23d) follows from the fact that, due to (3.23a), $\varepsilon^{1 / 2} s_{\varepsilon} \rightarrow^{*} 0$ in $L^{\infty}\left(0, T ; V_{0}^{*}\right)$. Moreover, by the definition of $\Psi_{\varepsilon}$ and by $(3.23 \mathrm{c}),(3.23 \mathrm{~d})$, and $(3.23 \mathrm{e})$, we immediately deduce that $\zeta=\eta$ a.e.. Finally, by compactness in the strong topology, from (3.23b) we can also deduce (at least) (cf. [31])

$$
\chi_{\varepsilon} \rightarrow \chi \quad \text { in } C^{0}([0, T] ; H) \cap L^{2}(0, T ; V) .
$$

Now, we are in the position of proving (3.5), due to the (weak) l.s.c. of norms and $J_{V}^{*}$, the above weak convergence results. Indeed, we can add (3.15) and (3.16) written for $\varepsilon$, integrate over $(0, t)$ (see $(3.17)$ ) and using lower semicontinuity arguments.

To prove that, under the additional Hyp. 2.4 (with $(A 1)$ or $(A 2)$ ) and the additional regularity for $s_{0},(s, \chi)$ is a weak solution to the original problem, we proceed as follows. We first point out that $\delta \in \partial_{V^{\prime}, V} J_{3}^{*}(\theta)$ (here the subdifferential is taken with respect to $\theta=s-\chi)$ belongs to $\partial_{V^{\prime}, V}^{s} J_{3}^{*}(s-\chi)$ as well as $-\delta \in V^{\prime}$ is identified with an element of $\partial_{V, V^{\prime}}^{\chi} J_{3}^{*}(s-\chi)$. Then, we recall the result by [6, Corollary 2.11, p. 41] ensuring that under our assumptions on the domains of the $J_{i}^{*}$ functions, the subdifferential of the sum of the three functions is given by the sum of the subdifferentials. 
Thus, we consider equations (2.18)-(2.19) in which we can split the subdifferentials as follows. First, we write

$$
\xi_{\varepsilon}=\xi_{\varepsilon}^{A}+\xi_{\varepsilon}^{B}
$$

where $\xi_{\varepsilon}^{A} \in \partial_{V, V^{\prime}} J_{2}^{*}\left(\chi_{\varepsilon}\right)$ and $\xi_{\varepsilon}^{B}=-\delta_{\varepsilon}$ in $V^{\prime}, \delta_{\varepsilon} \in \partial_{V^{\prime}, V} J_{3}^{*}\left(\theta_{\varepsilon}\right)$ (here the subdifferential is taken with respect to $\left.\theta_{\varepsilon}=s_{\varepsilon}-\chi_{\varepsilon}\right)$. Analogously, we have that

$$
\eta_{\varepsilon}=\eta_{\varepsilon}^{A}+\eta_{\varepsilon}^{B}
$$

where $\eta_{\varepsilon}^{A} \in \partial_{V^{\prime}, V} J_{1}^{*}\left(s_{\varepsilon}\right), \eta_{\varepsilon}^{B}=\delta_{\varepsilon} \in \partial_{V^{\prime}, V} J_{3}^{*}\left(\theta_{\varepsilon}\right)$, with $\theta_{\varepsilon}=s_{\varepsilon}-\chi_{\varepsilon}$.

Let us first assume that that $(A 2)$ holds. In this case, we have that $\eta_{\varepsilon}^{A}=0$. We can test the equation (3.6) by $\left(s_{\varepsilon}-\chi_{\varepsilon}\right)$ and integrate over $(0, t)$. Standard monotonicity arguments and the definition of sub-differential show that

$$
\left\|s_{\varepsilon}-\chi_{\varepsilon}\right\|_{L^{\infty}(0, T ; H)} \leqslant c
$$

and thus (see (3.19))

$$
\left\|s_{\varepsilon}\right\|_{L^{\infty}(0, T ; H)} \leqslant c
$$

Note that here, we have to use the further regularity required for $s_{0} \in H$. By compactness, we can deduce (see (3.23a)) that

$$
s_{\varepsilon} \rightarrow s \quad \text { in } C^{0}\left([0, T] ; V^{\prime}\right) .
$$

After observing that $\theta_{\varepsilon}=s_{\varepsilon}-\chi_{\varepsilon}$ strongly converges to $\theta=s-\chi$ in $C^{0}\left([0, T] ; V^{\prime}\right)$ (see (3.27) and (3.24)), we can combine this result with (3.23b), so that, exploiting semicontinuity arguments, it is a standard matter to get that $\eta \in \partial_{V^{\prime}, V} J_{3}^{*}(s-\chi)$ (also recall that $\zeta=\eta)$. Now, it remains to identify the limit $\xi$ of $\xi_{\varepsilon}$. We observe that $\xi_{\varepsilon}^{B} \rightarrow \xi^{B}=-\delta$ in $L^{2}\left(0, T ; V^{\prime}\right)$, and that we have already identified the weak limit. Now, we have to identify the limit of $\xi_{\varepsilon}^{A} \rightarrow \xi^{A}=\xi-\xi^{B}$ in $L^{2}\left(0, T ; V^{\prime}\right)$. Note that it is bounded in $L^{2}\left(0, T ; V^{\prime}\right)$ due to $(3.22)$ and $(3.21)$, where $\eta_{\varepsilon}=\eta_{\varepsilon}^{B}=-\xi_{\varepsilon}^{B}$ in $V^{\prime}$. To this aim, we use semicontinuity arguments for maximal monotone operators combining (3.24) with (3.23f).

Now, we consider the case when $(A 1)$ is assumed. Let us point out that in this case we cannot perform the estimate by $s_{\varepsilon}-\chi_{\varepsilon}$ (which actually improves the regularity of $s$ ). Thus, we proceed proving directly the identification of the limit functions, using the definition of sub-differential. Considering (3.21) and (3.19) one can deduce that

$$
\left\|\xi_{\varepsilon}^{B}\right\|_{L^{2}\left(0, T ; V^{\prime}\right)} \leqslant c
$$

independently on $\varepsilon$, so that $\eta_{\varepsilon}^{B}=-\xi_{\varepsilon}^{B}$ is bounded, too in $L^{2}\left(0, T ; V^{\prime}\right)$. Then, we test (3.6) by $A^{-1}\left(s_{\varepsilon}-\chi_{\varepsilon}\right)$ and integrate over $(0, t)$. Using (3.23a), (3.23b), (3.24), and $(3.23 \mathrm{~d}),(3.23 \mathrm{e})$, we can deduce (by weak lower semicontinuity of norms and $J_{2}^{*}$ )

$$
\limsup _{\varepsilon \searrow 0} \int_{0}^{t}\left\langle s_{\varepsilon}-\chi_{\varepsilon}, \eta_{\varepsilon}^{B}\right\rangle \leqslant \int_{0}^{t}\left\langle s-\chi, \eta^{B}\right\rangle .
$$


Indeed, we have in particular that

$$
\limsup _{\varepsilon \searrow 0}-\int_{0}^{t} \int_{\Omega} \eta_{\varepsilon}^{A}\left(s_{\varepsilon}-\chi_{\varepsilon}\right)=-\liminf _{\varepsilon \searrow 0} \int_{0}^{t} \int_{\Omega} \eta_{\varepsilon}^{A} s_{\varepsilon}-\eta_{\varepsilon}^{A} \chi_{\varepsilon} \leqslant-\int_{0}^{t} \int_{\Omega} \eta^{A} s-\eta^{A} \chi
$$

due to weak semicontinuity of $J_{1}^{*}$ and (3.24). This allows us to identify $\eta^{B} \in \partial_{V^{\prime}, V}^{s}(s-$ $\chi)$. At this point we can test the same equation by $A^{-1} s_{\varepsilon}$, and by analogous argument (and exploiting the identification we have already proved) we get $\eta^{A} \in \partial_{V^{\prime}, V} J_{1}^{*}(s)$. The final identification $\xi^{A}$ easily follows by the boundedness of $\xi_{\varepsilon}^{A}$ in $L^{2}\left(0, T ; V^{\prime}\right)$ and (3.24) as we have detailed in the proof under the assumption $(A 2)$.

This concludes the proof of Theorem 3.1.

We are ready now to state the last result of our paper concerning uniqueness of solutions for problem (2.18-2.19), (3.1-3.2).

Theorem 3.4. Assume Hypotheses 2.2 and 2.3, 2.4 and take $\left(s_{0}, \chi_{0}\right) \in D\left(J_{V}^{*}\right)$, and suppose moreover that

the maps $\chi \mapsto \partial_{V^{\prime}, V}^{s} J_{V}^{*}(s, \chi)$ and $\chi \mapsto \partial_{V, V^{\prime}}^{\chi} J_{V}^{*}(s, \chi)$ are Lipschitz continuous

from $V$ to $V_{0}$ and from $V$ to $V^{\prime}$, respectively, for every $s \in V^{\prime}$. Then the solution $(s, \chi)$ of (2.18-2.19), (3.1-3.2) is uniquely determined and the following continuous dependence estimate holds true:

$$
\begin{array}{r}
\left\|\left(s_{1}-s_{2}\right)(t)\right\|_{V^{\prime}}^{2}+\left\|\left(\chi_{1}-\chi_{2}\right)(t)\right\|_{H}^{2}+\int_{0}^{t}\left\|\nabla\left(\chi_{1}-\chi_{2}\right)\right\|_{H}^{2} \mathrm{~d} \tau \leqslant \\
+\|\left(\left\|\left(s_{1}-s_{2}\right)(0)\right\|_{V^{\prime}}^{2}\right. \\
\left.+\left\|\left(\chi_{1}-\chi_{2}\right)(0)\right\|_{H}^{2}\right) .
\end{array}
$$

Proof. Let us take the difference of the two equations (2.18) and the two relations (2.19) corresponding to two different solutions $\left(s_{i}, \chi_{i}\right), i=1,2$ and test them by $A^{-1}\left(s_{1}-s_{2}\right)$ and $\left(\chi_{1}-\chi_{2}\right)$, respectively. Integrating over $(0, t)$, for $t \in[0, T]$, using Hyp. 2.8 and Hyp. 2.3, we get

$$
\begin{aligned}
& \left\|\left(s_{1}-s_{2}\right)(t)\right\|_{V^{\prime}}^{2}+\left\|\left(\chi_{1}-\chi_{2}\right)(t)\right\|_{H}^{2}+2 \int_{0}^{t}\left\|\nabla\left(\chi_{1}-\chi_{2}\right)\right\|_{H}^{2} \mathrm{~d} \tau \leqslant\left\|\left(s_{1}-s_{2}\right)(0)\right\|_{V^{\prime}}^{2} \\
& +\left\|\left(\chi_{1}-\chi_{2}\right)(0)\right\|_{H}^{2}-2 \int_{0}^{t}\left(\gamma\left(\chi_{1}\right)-\gamma\left(\chi_{2}\right), \chi_{1}-\chi_{2}\right) \mathrm{d} \tau \\
& \quad-2 \int_{0}^{t}\left\langle s_{1}-s_{2}, \partial_{V^{\prime}, V}^{s} J_{V}^{*}\left(s_{2}, \chi_{1}\right)-\partial_{V^{\prime}, V}^{s} J_{V}^{*}\left(s_{2}, \chi_{2}\right)\right\rangle \mathrm{d} \tau \\
& \quad-2 \int_{0}^{t}\left(\chi_{1}-\chi_{2}, \partial_{V, V^{\prime}}^{\chi} J_{V}^{*}\left(s_{2}, \chi_{1}\right)-\partial_{V, V^{\prime}}^{\chi} J_{V}^{*}\left(s_{2}, \chi_{2}\right)\right) \mathrm{d} \tau .
\end{aligned}
$$

Using then the Lipschitz continuity of $\gamma$ and assumption (3.30), together with a standard Gronwall lemma, we obtain exactly (3.31). 
Remark 3.5. Let us mention that a possible application for Theorem 3.4 consists of the Caginalp phase-field model introduced in Section 2.3 (cf. also [7]). Moreover the Theorem applies to the case of quite standard approximations of the general nonlinear phase-field systems where the monotone nonlinearities are replaced by their Lipschitzcontinuous Yosida approximations.

\section{Acknowledgements}

The financial support of the FP7-IDEAS-ERC-StG \#256872 (EntroPhase) is gratefully acknowledged by the authors. The present paper also benefits from the support of the MIUR-PRIN Grant 2010A2TFX2 "Calculus of Variations" for EB, the GNAMPA (Gruppo Nazionale per l'Analisi Matematica, la Probabilità e le loro Applicazioni) of INdAM (Istituto Nazionale di Alta Matematica), and the IMATI - C.N.R. Pavia for EB and ER. The authors would also like to thank Riccarda Rossi for the fruitful discussions on the topic and the anonymous referees for their precious comments that helped us to improve the paper's results.

\section{References}

[1] V. Barbu: Nonlinear Semigroups and Differential Equations in Banach Spaces, Noordhoff, Leyden (1976).

[2] V. Barbu, P. Colli, G. Gilardi, M. Grasselli: Existence, uniqueness, and longtime behavior for a nonlinear Volterra integrodifferential equation, Differential Integral Equations, 13 no. 10-12, 1233-1262 (2000).

[3] E. Bonetti, P. Colli, M. Frémond: A phase field model with thermal memory governed by the entropy balance, Math. Models Methods Appl. Sci., 13 no. 11, 1565-1588 (2003).

[4] E. Bonetti, M. Frémond: A phase transition model with the entropy balance: Math. Meth. Appl. Sci., 26, 539-556 (2003).

[5] E. Bonetti, M. Frémond, E. Rocca: A new dual approach for a class of phase transitions with memory: existence and long-time behaviour of solutions, J. Math. Pure Appl., 88 (2007), 455-481.

[6] H. Brezis: Opérateurs Maximaux Monotones et Semi-groupes de Contractions dans les Espaces de Hilbert, North-Holland Math. Studies, 5, North-Holland, Amsterdam (1973).

[7] G. Caginalp, The dynamics of a conserved phase-field system: Stefan-like, HeleShow, and Cahn-Hilliard models as asymptotic limits, IMA J. Appl. Math. 44 (1990), 77-94.

[8] J. Cahn, J. Hilliard, Free energy of a nonuniform system. I. Interfacial free energy, J. Chem. Phys. 28 (1958), 258-267. 
[9] P. Colli, P. Krejčí, E. Rocca, J. Sprekels: Nonlinear evolution inclusions arising from phase change models, Czech. Math. J., 57 (2007), 1067-1098.

[10] P. Colli, A. Visintin On a class of doubly nonlinear evolution equations, Comm. Partial Differential Equations, 15 (1990), 737-756.

[11] M. Fabrizio, C. Giorgi: Sulla termodinamica dei materiali semplici, Boll. UMI 6 (1986), 441-464.

[12] E. Feireisl, H. Petzeltová, E. Rocca: Existence of solutions to a phase transition model with microscopic movements, Math. Methods Appl. Sci. 32 (2009), 13451369.

[13] M. Frémond: Nonsmooth thermomechanics, Springer-Verlag, Berlin (2002).

[14] A.E. Green, P.M. Naghdi: A re-examination of the basic postulates of thermomechanics, Proc. R. Soc. Lond. A 432 (1991), 171-194.

[15] A.E. Green, P.M. Naghdi: A demonstration of consistency of an entropy balance with balance of energy, ZAMP 42 (1991), 159-168

[16] M.E. Gurtin: Generalized Ginzburg-Landau and Cahn-Hilliard equations based on a microforce balance, Physica D 92 (1996), 178-192.

[17] A. Hawkins-Daruud, K. G. van der Zee, J. T. Oden: Numerical simulation of a thermodynamically consistent four-species tumor growth model, Int. J. Numer. Math. Biomed. Engng. 28 (2011), 3-24.

[18] D. Hilhorst, J. Kampmann, T. N. Nguyen, K. G. van der Zee: Formal asymptotic limit of a diffuse-interface tumor-growth model, Math. Models Methods Appl. Sci. 25 (2015), 1011-1043.

[19] F. Luterotti, G. Schimperna, U. Stefanelli: Global solution to a phase field model with irreversible and constrained phase evolution, Quart. Appl. Math. 60 (2002), $301-316$.

[20] A. Mielke: Thermomechanical modeling of energy-reaction-diffusion systems, including bulk-interface interactions, Discrete Contin. Dyn. Syst. Ser. S, 6 (2013), 479-499.

[21] A. Mielke, R. Rossi, G. Savaré: Nonsmooth analysis of doubly nonlinear evolution equations, Calc. Var. Partial Differential Equations, 46 (2013), 253-310.

[22] A. Mielke: Free energy, free entropy, and a gradient structure for thermoplasticity, preprint WIAS n. 2091 (2015).

[23] A. Mielke, F. Theil: On rate-independent hysteresis models, Nonlinear Diff. Eq. Appl. 11 (2004), 151-189.

[24] A. Miranville, G. Schimperna: Global solution to a phase transition model based on a microforce balance, J. Evol. Equ. 5 (2005), 253-276. 
[25] O. Penrose, P.C. Fife: Thermodynamically consistent models of phase field type for the kinetics of phase transitions, Phys. D, 43, 44-62 (1990).

[26] P. Podio-Guidugli: A virtual power format for thermomechanics, Continuum Mech. Thermodyn. 20 (2009), 479-487.

[27] E. Rocca, R. Scala: A rigorous sharp interface limit of a diffuse interface model related to tumor growth, preprint arXiv:1606.04663 (2016), 1-24.

[28] R. Rossi, G. Savarè: Existence and approximation results for gradient flows, Atti Accad. Naz. Lincei Cl. Sci. Fis. Mat. Natur. Rend. Lincei (9) Mat. Appl., 15 (2004), 183-196.

[29] R. Rossi, G. Savaré: Gradient flows of non convex functionals in Hilbert spaces and applications, ESAIM Control Optim. Calc. Var. 12 (2006), 564-614.

[30] G. Schimperna, A. Segatti, S. Zelik: Asymptotic uniform boundedness of energy solutions to the Penrose-Fife model, J. Evol. Equ. 12 (2012), 863-890.

[31] J. Simon: Compact sets in the space $L^{p}(0, T ; B)$, Ann. Mat. Pura Appl. (4), 146, 65-96 (1987). 